\title{
METHOD OF FUNDAMENTAL SOLUTIONS FOR PLATE VIBRATIONS IN MULTIPLY CONNECTED DOMAINS
}

\author{
C. C. Tsai * \\ Department of Information Technology \\ Toko University \\ Chia-Yi County, Taiwan 61363, R.O.C. \\ D. L. Young ${ }^{* * *}$ C. M. Fan ${ }^{* * * *}$ \\ Department of Civil Engineering and Hydrotech Research Institute \\ National Taiwan University \\ Taipei, Taiwan 10617, R.O.C.
}

\begin{abstract}
This paper develops the method of fundamental solutions (MFS) to solve eigenfrequencies of plate vibrations of multiply connected domains. The complex-valued MFS combined with the mix potential method are utilized in order to avoid the spurious eigenvalues. The benchmarked problems of annular plates with clamped, simply supported and free boundary conditions are studied analytically as well as numerically. Wherein the results demonstrate that all true eigenvalues are contained and no spurious eigenvalues are included. In the analytical studies, the continuous version of the MFS is utilized to obtain the eigenequation by applying the degenerate kernels and Fourier series. The proposed numerical method is free from singularities, meshes, and numerical integrations and thus can be easily utilized to solve plate vibrations free from spurious eigenvalues in multiply connected domains.
\end{abstract}

Keywords : Method of fundamental solutions, Mix potential method, Plate vibration, Spurious eigenvalues, Annular plate, Clamped, Simply supported, Free boundary conditions.

\section{INTRODUCTION}

Meshless numerical methods have recently become alternatives to the classical mesh dependent numerical methods, such as the finite difference method (FDM), finite element method (FEM), and boundary element method (BEM). The MFS, first developed by $\mathrm{Ku}-$ pradze and Aleksidze [1] in 1964, has re-emerged as a promising meshless numerical scheme for solving various types of partial differential equations. The basic idea of the MFS is to decompose the solutions of the partial differential equations into a linear combination of the fundamental solutions, in which source points are located on a fictitious boundary outside the computational domain. Here, the intensities of the sources are the unknown parameters to be found. Excellent reviews of the MFS are available in the recent literature [2 5]

For time dependent problems, they can be treated either by time marching [6 8] or by eigenmode expansion [9], where the later one is considered for plate problems in this paper. There are many numerical methods available in the analysis of plate vibrations. Leissa et al. [10] has provided a comprehensive survey for the numerical solutions of plate vibration problems. Integral equations and BEM have also been utilized to solve plate vibration problems for a long time. Kitahara [11] employed the complex-valued boundary integral equation (BIE) method to solve the eigenvalues and eigenmodes for plate vibrations with various boundary conditions. On the other hand, Hutchinson [12] has carried out a series of studies by utilizing the BIE with real-part kernel to solve plate vibration problems. However, he did not circumvent the problems of spurious eigenvalues. Recently, Chen et al. [13] further developed this method and utilized the SVD updating technique to avoid the occurrence of the spurious eigenvalues. However, a general method for computing eigenvalues with various boundary conditions in multiply connected domains, especially for a simple scheme free from spurious eigenvalues, is still lacking.

The MFS, which is highly related to the BEM, has also been applied to solve eigenvalue problems [4].

\footnotetext{
* Assistant Professor ** Professor ***Postdoctoral Fellow
} 
Kondapalli and Shippy [14] first applied the MFS for acoustic scattering. Recently, Kang and Lee [15] employed the non-dimensional dynamic influence function (NDIF) method to solve the eigenproblem of an acoustic cavity. Chen et al. [16] commented that the NDIF method is a special case of the MFS with imaginary-part kernel and later further revisited this method in a series of works [17,18]. Later, Kang and Lee [19] extended the NDIF for plate vibrations with clamped boundary condition. On the other hand, Karageorghis [20], Tasi et al. [9], Young et al. [21] and Chen et al. [22] all applied the complex-valued MFS for 2D Helmholtz problem in simply connected domains.

To avoid the fictitious eigenvalues of the exterior acoustical problems, Burton and Miller [23] have successfully applied the combination of the single- and double-layer potentials for direct BIE. On the other hand, it was shown independently by Panich [24] and by Brakhage \& Werner [25] that the mix potential method for indirect BIE is also feasible. In simply connected domains, there are no spurious eigenvalues if the complex-valued BIE is employed [26]. However, the spurious eigenvalues always appear when the complexvalued BIE is employed to the multiply connected problem [11,27]. Therefore, Chen et al. [27] and Tsai et al. [9] applied the mix potential method to deal with the acoustical problems in multiply connected domains successfully.

In this paper, we will develop the complex-valued MFS combined with the mix potential method to obtain eigenfrequencies of thin plate vibrations with the clamped, simply supported and free boundary conditions for multiply connected domains. The free vibration analysis of an annular plate is investigated both analytically and numerically using the discrete and continuous versions of the MFS schemes. It is found that all true eigenvalues are captured and no spurious eigenvalues are contaminated by using the proposed complex-valued MFS combined with the mix potential method. The paper is organized as follows. In Section 2 the MFS formulations are introduced. The analytical derivations are carried out in Section 3. The numerical results are presented in Section 4. The final conclusions drawn based on the present study are given in Section 5.

\section{MFS FORMULATION IN MULTIPLY CONNECTED DOMAIN}

For free flexural vibration of a uniform thin plate in a multiply connected domain (Fig. 1), the governing equation and boundary conditions are [11]:

$$
\begin{cases}\nabla^{4} u(\mathbf{x})-\lambda^{4} u(\mathbf{x})=0 & \mathbf{x} \in \Omega \\ B_{1}^{I} u(\mathbf{x})=B_{2}^{I} u(\mathbf{x})=0 & \mathbf{x} \in \Gamma^{I} \\ B_{1}^{E} u(\mathbf{x})=B_{2}^{E} u(\mathbf{x})=0 & \mathbf{x} \in \Gamma^{E}\end{cases}
$$

where $u(x)$ is the function of lateral displacement,

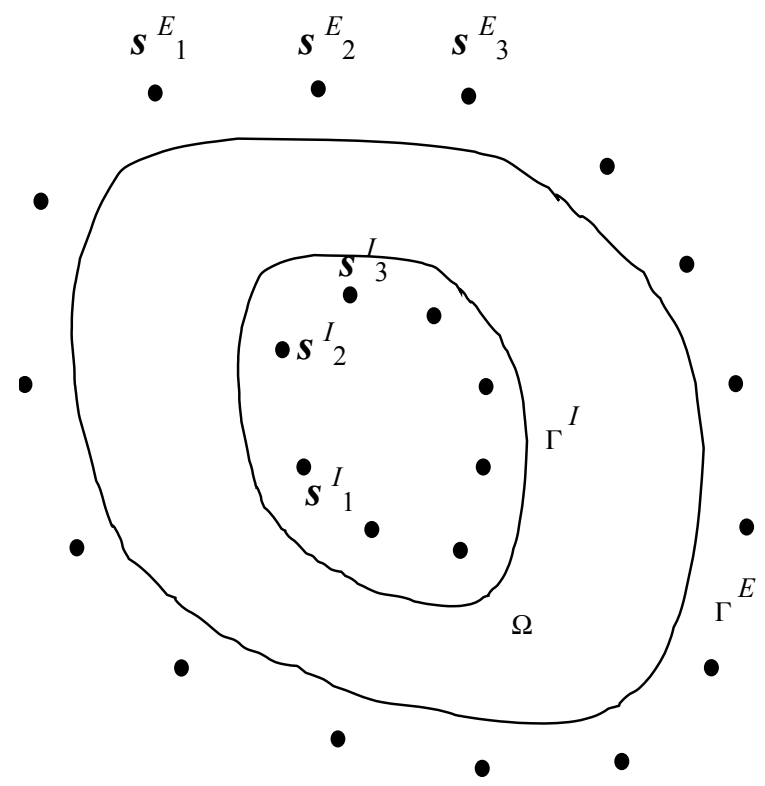

Fig. 1 Geometry configuration of the MFS for plate vibrations in a multiply connected domain

$\lambda^{4}=\frac{\omega^{2} \rho_{s}}{D}$ is the frequency parameter, $\omega$ is the circular frequency, $\rho_{s}$ is the mass density per unit area, and $h$ is the plate thickness. Also, $D=\frac{E h^{3}}{12\left(1-v^{2}\right)}$ is the flexural rigidity of the thin plate, $v$ is the Poisson ratio, $E$ is the Young's modulus and $\Omega$ is the domain of interest with interior boundary $\Gamma^{I}$ and exterior boundary $\Gamma^{E}$. In Eq. (1), Both $B_{1}^{I} \quad \& \quad B_{2}^{I}$ and $B_{1}^{E} \& B_{2}^{E}$ are any two of the following boundary operators:

$$
\begin{aligned}
& \mathbf{K}_{u}(\bullet)=1 \\
& \mathbf{K}_{\theta}(\bullet)=\frac{\partial(\bullet)}{\partial n_{\mathbf{x}}} \\
& \mathbf{K}_{m}(\bullet)=v \nabla_{\mathbf{x}}^{2}(\bullet)+(1-v) \frac{\partial^{2}(\bullet)}{\partial n_{\mathbf{x}}^{2}} \\
& \mathbf{K}_{v}(\bullet)=\frac{\partial \nabla_{\mathbf{x}}^{2}(\bullet)}{\partial n_{\mathbf{x}}}+(1-v) \frac{\partial}{\partial t_{\mathbf{x}}} \frac{\partial^{2}(\bullet)}{\partial n_{\mathbf{x}} \partial t_{\mathbf{x}}}
\end{aligned}
$$

where $\frac{\partial}{\partial n_{\mathbf{x}}}$ and $\frac{\partial}{\partial t_{\mathbf{x}}}$ are the normal and tangential derivatives, respectively, on the boundary point $\mathbf{x}$. In the above equations, we denote $\mathbf{K}_{u}(u(\mathbf{x})), \mathbf{K}_{\theta}(u(\mathbf{x}))$, $\mathbf{K}_{m}(u(\mathbf{x}))$, and $\mathbf{K}_{v}(u(\mathbf{x}))$ the lateral displacement, slope, normal moment, and effective sheer force respectively. Therefore, (2a) \& (2b) are selected for plate vibrations with clamped boundary condition, (2a) \& (2c) for simply supported boundary condition, and (2c) \& (2d) for free boundary condition.

The fundamental solution of the Eq. (1) is defined by

$$
\nabla^{4} G_{\lambda}(\mathbf{x} ; \mathbf{s})-\lambda^{4} G_{\lambda}(\mathbf{x} ; \mathbf{s})=-\delta(\mathbf{x}-\mathbf{s})
$$


where $\mathbf{x}$ are the coordinates of boundary field points and $\mathbf{s}$ are the coordinates of source points. Then, the fundamental solution is given by [11]:

$$
G_{\lambda}(\mathbf{x} ; \mathbf{s})=\frac{1}{8 \lambda^{2}}\left[-i H_{0}^{(1)}(\lambda r)+\frac{2}{\pi} K_{0}(\lambda r)\right]
$$

where $H_{0}^{(1)}(\lambda r)$ is the Hankel function of the first kind of order zero, and $K_{0}(\lambda r)$ is the modified Bessel function of the second kind of order zero, $r=|\mathbf{x}-\mathbf{s}|$ and $i$ is the image unit.

In the MFS formulation for multiply connected domain, the solution is assumed to be

$$
\begin{aligned}
u(\mathbf{x})= & \sum_{j=1}^{N^{E}}\left[\alpha_{j}^{E} U_{1, \lambda}\left(\mathbf{x}, \mathbf{s}_{j}^{E}\right)+\beta_{j}^{E} U_{2, \lambda}\left(\mathbf{x}, \mathbf{s}_{j}^{E}\right)\right] \\
& +\sum_{j=1}^{N^{I}}\left[\alpha_{j}^{I} U_{3, \lambda}\left(\mathbf{x}, \mathbf{s}_{j}^{I}\right)+\beta_{j}^{I} U_{4, \lambda}\left(\mathbf{x}, \mathbf{s}_{j}^{I}\right)\right]
\end{aligned}
$$

where $\left\{\alpha_{j}^{E}, \beta_{j}^{E}\right\}$ and $\left\{\alpha_{j}^{I}, \beta_{j}^{I}\right\}$ are the intensities of the sources at $N^{E}$ exterior source points $\mathbf{s}_{j}^{E}$ and $N^{I}$ interior source points $\mathbf{s}_{j}^{I}$, respectively as depicted in Fig. 1. Here, the third and fourth terms are included to circumvent the spurious eigenvalues [9,23 25]. In Eq. (5), the kernels are defined by:

$$
\begin{aligned}
& U_{1, \lambda}(\mathbf{x}, \mathbf{s})=H_{0}^{(1)}(\lambda r) \\
& U_{2, \lambda}(\mathbf{x}, \mathbf{s})=K_{0}(\lambda r) \\
& U_{3, \lambda}(\mathbf{x}, \mathbf{s})=U_{1, \lambda}(\mathbf{x}, \mathbf{s})+i \frac{\partial U_{1, \lambda}(\mathbf{x}, \mathbf{s})}{\partial n_{\mathbf{s}}} \\
& U_{4, \lambda}(\mathbf{x}, \mathbf{s})=U_{2, \lambda}(\mathbf{x}, \mathbf{s})+i \frac{\partial U_{2, \lambda}(\mathbf{x}, \mathbf{s})}{\partial n_{\mathbf{s}}}
\end{aligned}
$$

Therefore, we have the following definitions for the corresponding kernels of other boundary conditions

$$
\begin{gathered}
\Theta_{i, \lambda}(\mathbf{x}, \mathbf{s})=\mathbf{K}_{\theta}\left(U_{i, \lambda}(\mathbf{x}, \mathbf{s})\right) \quad i=1, \cdots, 4 \\
M_{i, \lambda}(\mathbf{x}, \mathbf{s})=\mathbf{K}_{m}\left(U_{i, \lambda}(\mathbf{x}, \mathbf{s})\right) \quad i=1, \cdots, 4 \\
V_{i, \lambda}(\mathbf{x}, \mathbf{s})=\mathbf{K}_{v}\left(U_{i, \lambda}(\mathbf{x}, \mathbf{s})\right) \quad i=1, \cdots, 4
\end{gathered}
$$

In order to obtain $\left\{\alpha_{j}^{E}, \beta_{j}^{E}\right\}$ and $\left\{\alpha_{j}^{I}, \beta_{j}^{I}\right\}$, a collocation procedure is applied at $N=N^{I}+N^{E}$ boundary field points, which is formally adopted in the MFS [2 5]. That is,

$$
\begin{aligned}
\mathbf{K}_{u}\left(u\left(\mathbf{x}_{i}\right)\right)= & \sum_{j=1}^{N^{E}}\left[\alpha_{j}^{E} U_{1, \lambda}\left(\mathbf{x}_{i}, \mathbf{s}_{j}^{E}\right)+\beta_{j}^{E} U_{2, \lambda}\left(\mathbf{x}_{i}, \mathbf{s}_{j}^{E}\right)\right] \\
& +\sum_{j=1}^{N^{I}}\left[\alpha_{j}^{I} U_{3, \lambda}\left(\mathbf{x}_{i}, \mathbf{s}_{j}^{I}\right)+\beta_{j}^{I} U_{4, \lambda}\left(\mathbf{x}_{i}, \mathbf{s}_{j}^{I}\right)\right]=0 \\
& \text { for }\left\{\mathbf{x}_{i}\right\}_{1}^{N} \in \Gamma^{I}+\Gamma^{E}
\end{aligned}
$$

$$
\begin{aligned}
& \mathbf{K}_{\theta}\left(u\left(\mathbf{x}_{i}\right)\right)= \sum_{j=1}^{N^{E}}\left[\alpha_{j}^{E} \Theta_{1, \lambda}\left(\mathbf{x}_{i}, \mathbf{s}_{j}^{E}\right)+\beta_{j}^{E} \Theta_{2, \lambda}\left(\mathbf{x}_{i}, \mathbf{s}_{j}^{E}\right)\right] \\
&+ \sum_{j=1}^{N^{I}}\left[\alpha_{j}^{I} \Theta_{3, \lambda}\left(\mathbf{x}_{i}, \mathbf{s}_{j}^{I}\right)+\beta_{j}^{I} \Theta_{4, \lambda}\left(\mathbf{x}_{i}, \mathbf{s}_{j}^{I}\right)\right]=0 \\
& \text { for }\left\{\mathbf{x}_{i}\right\}_{1}^{N} \in \Gamma^{I}+\Gamma^{E} \\
& \mathbf{K}_{m}\left(u\left(\mathbf{x}_{i}\right)\right)= \sum_{j=1}^{N^{E}}\left[\alpha_{j}^{E} M_{1, \lambda}\left(\mathbf{x}_{i}, \mathbf{s}_{j}^{E}\right)+\beta_{j}^{E} M_{2, \lambda}\left(\mathbf{x}_{i}, \mathbf{s}_{j}^{E}\right)\right] \\
&+\sum_{j=1}^{N^{I}}\left[\alpha_{j}^{I} M_{3, \lambda}\left(\mathbf{x}_{i}, \mathbf{s}_{j}^{I}\right)+\beta_{j}^{I} M_{4, \lambda}\left(\mathbf{x}_{i}, \mathbf{s}_{j}^{I}\right)\right]=0 \\
& \text { for }\left\{\mathbf{x}_{i}\right\}_{1}^{N} \in \Gamma^{I}+\Gamma^{E} \\
&+\sum_{j=1}^{N^{I}}\left[\alpha_{j}^{I} V_{3, \lambda}\left(\mathbf{x}_{i}, \mathbf{s}_{j}^{I}\right)+\beta_{j}^{I} V_{4, \lambda}\left(\mathbf{x}_{i}, \mathbf{s}_{j}^{I}\right)\right]=0 \\
& \mathbf{K}_{v}\left(u\left(\mathbf{x}_{i}\right)\right)= \sum_{j=1}^{N^{E}}\left[\alpha_{j}^{E} V_{1, \lambda}\left(\mathbf{x}_{i}, \mathbf{s}_{j}^{E}\right)+\beta_{j}^{E} V_{2, \lambda}\left(\mathbf{x}_{i}, \mathbf{s}_{j}^{E}\right)\right] \\
& \text { for }\left\{\mathbf{x}_{i}\right\}_{1}^{N} \in \Gamma^{I}+\Gamma^{E}
\end{aligned}
$$

where $\left\{\mathbf{x}_{i}\right\}_{1}^{N}$ are the collocated boundary field points. For simplicity, we assume $\mathbf{s}_{j}^{E} \& \mathbf{s}_{j}^{I}$ to be a priori distributed source points and the number of collocated boundary field point is equal to the number of source points. This results in a $2 N \times 2 N$ linear system;
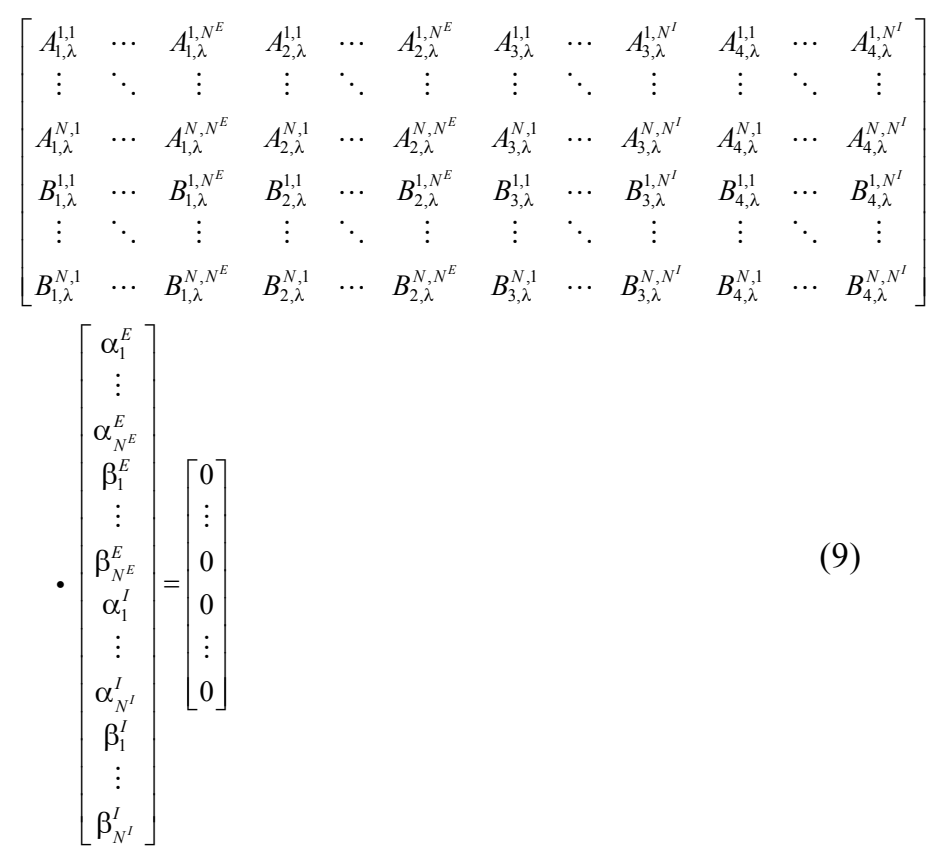

where

$A_{1, \lambda}^{i, j}=U_{1, \lambda}\left(\mathbf{x}_{i}, \mathbf{s}_{j}^{E}\right), A_{2, \lambda}^{i, j}=U_{2, \lambda}\left(\mathbf{x}_{i}, \mathbf{s}_{j}^{E}\right), A_{3, \lambda}^{i, j}=U_{3, \lambda}\left(\mathbf{x}_{i}, \mathbf{s}_{j}^{I}\right)$, $A_{4, \lambda}^{i, j}=U_{4, \lambda}\left(\mathbf{x}_{i}, \mathbf{s}_{j}^{I}\right), B_{1, \lambda}^{i, j}=\Theta_{1, \lambda}\left(\mathbf{x}_{i}, \mathbf{s}_{j}^{E}\right), B_{2, \lambda}^{i, j}=\Theta_{2, \lambda}\left(\mathbf{x}_{i}, \mathbf{s}_{j}^{E}\right)$, $B_{3, \lambda}^{i, j}=\Theta_{3, \lambda}\left(\mathbf{x}_{i}, \mathbf{s}_{j}^{I}\right)$, and $B_{4, \lambda}^{i, j}=\Theta_{4, \lambda}\left(\mathbf{x}_{i}, \mathbf{s}_{j}^{I}\right)$ for clamped boundary condition, and similarly for other types of boundary conditions. Equation (9) is an eigenproblem for $\lambda$ and we are searching for eigenvalues $\lambda_{1}<\lambda_{2}<\lambda_{3}$ $<\cdots$ such that Eq. (9) has nontrivial solutions. 


\section{ANALYTIC SOLUTIONS}

In general, the eigenfrequencies of plate vibrations obtained by the MFS cannot be solved analytically except for some special geometric shapes. We therefore solve the following problems analytically by utilizing the continuous version of the MFS: (I) annular plate with clamped boundary condition, (II) annular plate with simply supported boundary condition and (III) annular plate with free boundary condition. In the following derivations, we assume the source points $\mathbf{s}_{j}^{E} \&$ $\mathbf{s}_{j}^{I}$ are dense enough so that the equations of the MFS, Eq. (8), can be transformed into the integral forms as follows:

$$
\begin{aligned}
\mathbf{K}_{u}(u(\mathbf{x}))= & \int\left[\alpha^{E}\left(\mathbf{s}^{E}\right) U_{1, \lambda}\left(\mathbf{x}, \mathbf{s}^{E}\right)+\beta^{E}\left(\mathbf{s}^{E}\right) U_{2, \lambda}\left(\mathbf{x}, \mathbf{s}^{E}\right)\right] d \Gamma^{E}\left(\mathbf{s}^{E}\right) \\
& +\int\left[\alpha^{I}\left(\mathbf{s}^{I}\right) U_{3, \lambda}\left(\mathbf{x}, \mathbf{s}^{I}\right)+\beta^{I}\left(\mathbf{s}^{I}\right) U_{4, \lambda}\left(\mathbf{x}, \mathbf{s}^{I}\right)\right] d \Gamma^{I}\left(\mathbf{s}^{I}\right)
\end{aligned}
$$

$\mathbf{K}_{\theta}(u(\mathbf{x}))=\int\left[\alpha^{E}\left(\mathbf{s}^{E}\right) \Theta_{1, \lambda}\left(\mathbf{x}, \mathbf{s}^{E}\right)+\beta^{E}\left(\mathbf{s}^{E}\right) \Theta_{2, \lambda}\left(\mathbf{x}, \mathbf{s}^{E}\right)\right] d \Gamma^{E}\left(\mathbf{s}^{E}\right)$ $+\int\left[\alpha^{I}\left(\mathbf{s}^{I}\right) \Theta_{3, \lambda}\left(\mathbf{x}, \mathbf{s}^{I}\right)+\beta^{I}\left(\mathbf{s}^{I}\right) \Theta_{4, \lambda}\left(\mathbf{x}, \mathbf{s}^{I}\right)\right] d \Gamma^{I}\left(\mathbf{s}^{I}\right)$

$$
\begin{aligned}
\mathbf{K}_{m}(u(\mathbf{x}))= & \int\left[\alpha^{E}\left(\mathbf{s}^{E}\right) M_{1, \lambda}\left(\mathbf{x}, \mathbf{s}^{E}\right)+\beta^{E}\left(\mathbf{s}^{E}\right) M_{2, \lambda}\left(\mathbf{x}, \mathbf{s}^{E}\right)\right] d \Gamma^{E}\left(\mathbf{s}^{E}\right) \\
& +\int\left[\alpha^{I}\left(\mathbf{s}^{I}\right) M_{3, \lambda}\left(\mathbf{x}, \mathbf{s}^{I}\right)+\beta^{I}\left(\mathbf{s}^{I}\right) M_{4, \lambda}\left(\mathbf{x}, \mathbf{s}^{I}\right)\right] d \Gamma^{I}\left(\mathbf{s}^{I}\right)
\end{aligned}
$$

$$
\begin{aligned}
\mathbf{K}_{v}(u(\mathbf{x}))= & \int\left[\alpha^{E}(\mathbf{s}) V_{1, \lambda}\left(\mathbf{x}, \mathbf{s}^{E}\right)+\beta^{E}\left(\mathbf{s}^{E}\right) V_{2, \lambda}\left(\mathbf{x}, \mathbf{s}^{E}\right)\right] d \Gamma^{E}\left(\mathbf{s}^{E}\right) \\
& +\int\left[\alpha^{I}\left(\mathbf{s}^{I}\right) V_{3, \lambda}\left(\mathbf{x}, \mathbf{s}^{I}\right)+\beta^{I}\left(\mathbf{s}^{I}\right) V_{4, \lambda}\left(\mathbf{x}, \mathbf{s}^{I}\right)\right] d \Gamma^{I}\left(\mathbf{s}^{I}\right)
\end{aligned}
$$

We consider the annular plate, as described in Fig. 2, and let $\mathbf{s}^{E}=\left(R^{E}, \theta^{E}\right), \mathbf{s}^{I}=\left(R^{I}, \theta^{I}\right)$, and $\mathbf{x}=(\rho, \phi)$ denote the interior source, exterior source, and boundary field points, respectively. To analytically derive the MFS solutions in an annular domain, it is necessary to decompose the kernels $(6 \mathrm{a}) \sim(6 \mathrm{~d})$ into circular harmonics, which will result in the following degenerate kernels $[9,13]$ :

$$
\begin{aligned}
U_{1, \lambda}\left(\mathbf{x}, \mathbf{s}^{E}\right)= & \sum_{m=-\infty}^{m=\infty} H_{m}^{(1)}\left(\lambda R^{E}\right) J_{m}(\lambda \rho) \cos \left(m\left(\theta^{E}-\phi\right)\right)(11 \\
U_{2, \lambda}\left(\mathbf{x}, \mathbf{s}^{E}\right)= & \sum_{m=-\infty}^{m=\infty} K_{m}\left(\lambda R^{E}\right) I_{m}(\lambda \rho) \cos \left(m\left(\theta^{E}-\phi\right)\right) \\
U_{3, \lambda}\left(\mathbf{x}, \mathbf{s}^{I}\right)= & \sum_{m=-\infty}^{m=\infty}\left[J_{m}\left(\lambda R^{I}\right)-i \lambda J_{m}^{\prime}\left(\lambda R^{I}\right)\right] \\
& \cdot H_{m}^{(1)}(\lambda \rho) \cos \left(m\left(\theta^{I}-\phi\right)\right) \\
U_{4, \lambda}\left(\mathbf{x}, \mathbf{s}^{I}\right)= & \sum_{m=-\infty}^{m=\infty}\left[I_{m}\left(\lambda R^{I}\right)-i k I_{m}^{\prime}\left(\lambda R^{I}\right)\right] \\
& \cdot K_{m}(\lambda \rho) \cos \left(m\left(\theta^{I}-\phi\right)\right)
\end{aligned}
$$

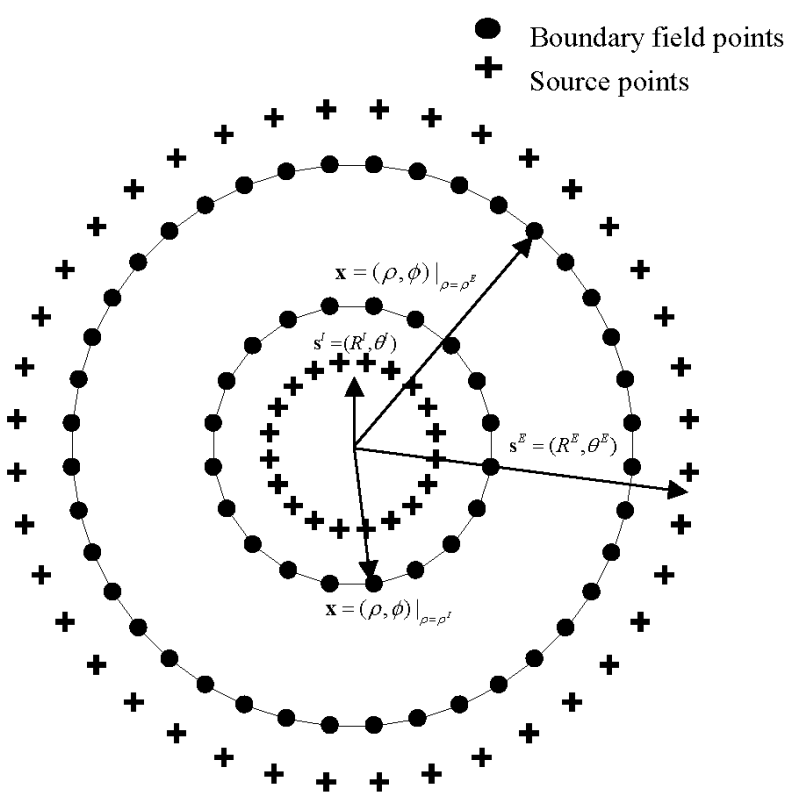

Fig. 2 Symbol notations of boundary field and source points for the MFS formulation in annular plate vibrations

where $J_{m}(\lambda \rho)$ is the Bessel function of the first kind of order $m, H_{m}^{(1)}(\lambda R)$ is the Hankel function of the first kind of order $m, I_{m}(\lambda \rho)$ is the modified Bessel function of the first kind of order $m$, and $K_{m}(\lambda \rho)$ is the modified Bessel function of the second kind of order $m$. Moreover, $R^{E}>\rho$ and $R^{I}<\rho$ are assumed in the above equations. Therefore, we have the following degenerate kernels for Eqs. $(7 a) \sim(7 c)$ :

$$
\Theta_{1, \lambda}\left(\mathbf{x}, \mathbf{s}^{E}\right)=\sum_{m=-\infty}^{m=\infty} H_{m}^{(1)}\left(\lambda R^{E}\right) \mathbf{K}_{\theta}\left[J_{m}(\lambda \rho) \cos \left(m\left(\theta^{E}-\phi\right)\right)\right]
$$

$\Theta_{2, \lambda}\left(\mathbf{x}, \mathbf{s}^{E}\right)=\sum_{m=-\infty}^{m=\infty} K_{m}\left(\lambda R^{E}\right) \mathbf{K}_{\theta}\left[I_{m}(\lambda \rho) \cos \left(m\left(\theta^{E}-\phi\right)\right)\right]$

$\Theta_{3, \lambda}\left(\mathbf{x}, \mathbf{s}^{I}\right)=\sum_{m=-\infty}^{m=\infty}\left[J_{m}\left(\lambda R^{I}\right)-i \lambda J_{m}^{\prime}\left(\lambda R^{I}\right)\right]$

$$
\text { - } \mathbf{K}_{\theta}\left[H_{m}^{(1)}(\lambda \rho) \cos \left(m\left(\theta^{I}-\phi\right)\right)\right]
$$

$\Theta_{4, \lambda}\left(\mathbf{x}, \mathbf{s}^{I}\right)=\sum_{m=-\infty}^{m=\infty}\left[I_{m}\left(\lambda R^{I}\right)-i k I_{m}^{\prime}\left(\lambda R^{I}\right)\right]$

$$
\text { - } \mathbf{K}_{\theta}\left[K_{m}(\lambda \rho) \cos \left(m\left(\theta^{I}-\phi\right)\right)\right]
$$

$M_{1, \lambda}\left(\mathbf{x}, \mathbf{s}^{E}\right)=\sum_{m=-\infty}^{m=\infty} H_{m}^{(1)}\left(\lambda R^{E}\right) \mathbf{K}_{m}\left[J_{m}(\lambda \rho) \cos \left(m\left(\theta^{E}-\phi\right)\right)\right]$

$$
M_{2, \lambda}\left(\mathbf{x}, \mathbf{s}^{E}\right)=\sum_{m=-\infty}^{m=\infty} K_{m}\left(\lambda R^{E}\right) \mathbf{K}_{m}\left[I_{m}(\lambda \rho) \cos \left(m\left(\theta^{E}-\phi\right)\right)\right]
$$




$$
\begin{aligned}
M_{3, \lambda}\left(\mathbf{x}, \mathbf{s}^{I}\right)= & \sum_{m=-\infty}^{m=\infty}\left[J_{m}\left(\lambda R^{I}\right)-i \lambda J_{m}{ }^{\prime}\left(\lambda R^{I}\right)\right] \\
& \cdot \mathbf{K}_{m}\left[H_{m}^{(1)}(\lambda \rho) \cos \left(m\left(\theta^{I}-\phi\right)\right)\right] \quad(12 \mathrm{~g}) \\
M_{4, \lambda}\left(\mathbf{x}, \mathbf{s}^{I}\right)= & \sum_{m=-\infty}^{m=\infty}\left[I_{m}\left(\lambda R^{I}\right)-i k I_{m}{ }^{\prime}\left(\lambda R^{I}\right)\right] \\
& \cdot \mathbf{K}_{m}\left[K_{m}(\lambda \rho) \cos \left(m\left(\theta^{I}-\phi\right)\right)\right] \quad(12 \mathrm{~h}) \\
V_{1, \lambda}\left(\mathbf{x}, \mathbf{s}^{E}\right)= & \sum_{m=-\infty}^{m=\infty} H_{m}^{(1)}\left(\lambda R^{E}\right) \mathbf{K}_{v}\left[J_{m}(\lambda \rho) \cos \left(m\left(\theta^{E}-\phi\right)\right)\right] \\
V_{2, \lambda}\left(\mathbf{x}, \mathbf{s}^{E}\right)= & \sum_{m=-\infty}^{m=\infty} K_{m}\left(\lambda R^{E}\right) \mathbf{K}_{v}\left[I_{m}(\lambda \rho) \cos \left(m\left(\theta^{E}-\phi\right)\right)\right] \\
V_{3, \lambda}\left(\mathbf{x}, \mathbf{s}^{I}\right)= & \sum_{m=-\infty}^{m=\infty}\left[J_{m}\left(\lambda R^{I}\right)-i \lambda J_{m}{ }^{\prime}\left(\lambda R^{I}\right)\right] \\
& \cdot \mathbf{K}_{v}\left[H_{m}^{(1)}(\lambda \rho) \cos \left(m\left(\theta^{I}-\phi\right)\right)\right] \\
V_{4, \lambda}\left(\mathbf{x}, \mathbf{s}^{I}\right)= & \sum_{m=-\infty}^{m=\infty}\left[I_{m}\left(\lambda R^{I}\right)-i k I_{m}{ }^{\prime}\left(\lambda R^{I}\right)\right] \\
& \bullet \mathbf{K}_{v}\left[K_{m}(\lambda \rho) \cos \left(m\left(\theta^{I}-\phi\right)\right)\right]
\end{aligned}
$$

We likewise decompose the source intensities (in Eq. (10)) into the Fourier series

$$
\begin{aligned}
& \alpha^{E}\left(\mathbf{s}^{E}\right)=\sum_{n=-\infty}^{\infty} A_{n}^{E} \cos n \theta^{E}+B_{n}^{E} \sin n \theta^{E} \\
& \beta^{E}\left(\mathbf{s}^{E}\right)=\sum_{n=-\infty}^{\infty} C_{n}^{E} \cos n \theta^{E}+D_{n}^{E} \sin n \theta^{E} \\
& \alpha^{I}\left(\mathbf{s}^{I}\right)=\sum_{n=-\infty}^{\infty} A_{n}^{I} \cos n \theta^{I}+B_{n}^{I} \sin n \theta^{I} \\
& \beta^{I}\left(\mathbf{s}^{I}\right)=\sum_{n=-\infty}^{\infty} C_{n}^{I} \cos n \theta^{I}+D_{n}^{I} \sin n \theta^{I}
\end{aligned}
$$

Substituting Eqs. (11) (13) into the integral forms of Eq. (10), we then obtain:

$$
\begin{aligned}
\mathbf{K}_{u}(u(\mathbf{x}))= & \pi \sum_{m=-\infty}^{m=\infty} H_{m}^{(1)}\left(\lambda R^{E}\right)\left\{\mathbf{K}_{u}\left[J_{m}(\lambda \rho)\left(A_{m}^{E} \cos m \phi+B_{m}^{E} \sin m \phi\right)\right]\right. \\
& +\pi \sum_{m=-\infty}^{m=\infty} K_{m}\left(\lambda R^{E}\right)\left\{\mathbf{K}_{u}\left[I_{m}(\lambda \rho)\left(C_{m}^{E} \cos m \phi+D_{m}^{E} \sin m \phi\right)\right]\right. \\
& +\pi \sum_{m=-\infty}^{m=\infty}\left[J_{m}\left(\lambda R^{I}\right)-i \lambda J_{m}{ }^{\prime}\left(\lambda R^{I}\right)\right] \\
& \cdot \mathbf{K}_{u}\left[H_{m}^{(1)}(\lambda \rho)\left(A_{m}^{I} \cos m \phi+B_{m}^{I} \sin m \phi\right)\right] \\
& +\pi \sum_{m=-\infty}^{m=\infty}\left[I_{m}\left(\lambda R^{I}\right)-i k I_{m}{ }^{\prime}\left(\lambda R^{I}\right)\right] \\
& \cdot \mathbf{K}_{u}\left[K_{m}(\lambda \rho)\left(C_{m}^{I} \cos m \phi+D_{m}^{I} \sin m \phi\right)\right]
\end{aligned}
$$

$$
\begin{aligned}
\mathbf{K}_{\theta}(u(\mathbf{x})) & =\pi \sum_{m=-\infty}^{m=\infty} H_{m}^{(1)}\left(\lambda R^{E}\right)\left\{\mathbf{K}_{\theta}\left[J_{m}(\lambda \rho)\left(A_{m}^{E} \cos m \phi+B_{m}^{E} \sin m \phi\right)\right]\right. \\
& +\pi \sum_{m=-\infty}^{m=\infty} K_{m}\left(\lambda R^{E}\right)\left\{\mathbf{K}_{\theta}\left[I_{m}(\lambda \rho)\left(C_{m}^{E} \cos m \phi+D_{m}^{E} \sin m \phi\right)\right]\right. \\
& +\pi \sum_{m=-\infty}^{m=\infty}\left[J_{m}\left(\lambda R^{I}\right)-i \lambda J_{m}^{\prime}\left(\lambda R^{I}\right)\right] \\
& \cdot \mathbf{K}_{\theta}\left[H_{m}^{(1)}(\lambda \rho)\left(A_{m}^{I} \cos m \phi+B_{m}^{I} \sin m \phi\right)\right] \\
& +\pi \sum_{m=-\infty}^{m=\infty}\left[I_{m}\left(\lambda R^{I}\right)-i k I_{m}^{\prime}\left(\lambda R^{I}\right)\right] \\
& \cdot \mathbf{K}_{\theta}\left[K_{m}(\lambda \rho)\left(C_{m}^{I} \cos m \phi+D_{m}^{I} \sin m \phi\right)\right]
\end{aligned}
$$

$\mathbf{K}_{m}(u(\mathbf{x}))=\pi \sum_{m=-\infty}^{m=\infty} H_{m}^{(1)}\left(\lambda R^{E}\right)\left\{\mathbf{K}_{m}\left[J_{m}(\lambda \rho)\left(A_{m}^{E} \cos m \phi+B_{m}^{E} \sin m \phi\right)\right]\right.$ $+\pi \sum_{m=-\infty}^{m=\infty} K_{m}\left(\lambda R^{E}\right)\left\{\mathbf{K}_{m}\left[I_{m}(\lambda \rho)\left(C_{m}^{E} \cos m \phi+D_{m}^{E} \sin m \phi\right)\right]\right.$$$
+\pi \sum_{m=-\infty}^{m=\infty}\left[J_{m}\left(\lambda R^{I}\right)-i \lambda J_{m}^{\prime}\left(\lambda R^{I}\right)\right]
$$$$
\text { - } \mathbf{K}_{m}\left[H_{m}^{(1)}(\lambda \rho)\left(A_{m}^{I} \cos m \phi+B_{m}^{I} \sin m \phi\right)\right]
$$$$
+\pi \sum_{m=-\infty}^{m=\infty}\left[I_{m}\left(\lambda R^{I}\right)-i k I_{m}^{\prime}\left(\lambda R^{I}\right)\right]
$$$$
\text { - } \mathbf{K}_{m}\left[K_{m}(\lambda \rho)\left(C_{m}^{I} \cos m \phi+D_{m}^{I} \sin m \phi\right)\right]
$$

$$
\begin{aligned}
\mathbf{K}_{v}(u(\mathbf{x}))= & \pi \sum_{m=-\infty}^{m=\infty} H_{m}^{(1)}\left(\lambda R^{E}\right)\left\{\mathbf{K}_{v}\left[J_{m}(\lambda \rho)\left(A_{m}^{E} \cos m \phi+B_{m}^{E} \sin m \phi\right)\right]\right. \\
& +\pi \sum_{m=-\infty}^{m=\infty} K_{m}\left(\lambda R^{E}\right)\left\{\mathbf{K}_{v}\left[I_{m}(\lambda \rho)\left(C_{m}^{E} \cos m \phi+D_{m}^{E} \sin m \phi\right)\right]\right. \\
& +\pi \sum_{m=-\infty}^{m=\infty}\left[J_{m}\left(\lambda R^{I}\right)-i \lambda J_{m}^{\prime}\left(\lambda R^{I}\right)\right] \\
& \cdot \mathbf{K}_{v}\left[H_{m}^{(1)}(\lambda \rho)\left(A_{m}^{I} \cos m \phi+B_{m}^{I} \sin m \phi\right)\right] \\
& +\pi \sum_{m=-\infty}^{m=\infty}\left[I_{m}\left(\lambda R^{I}\right)-i k I_{m}^{\prime}\left(\lambda R^{I}\right)\right] \\
& \cdot \mathbf{K}_{v}\left[K_{m}(\lambda \rho)\left(C_{m}^{I} \cos m \phi+D_{m}^{I} \sin m \phi\right)\right]
\end{aligned}
$$

in which the formula

$$
\cos (m(\theta-\phi)=\cos m \theta \cos m \phi+\sin m \theta \sin m \phi
$$

and the following orthogonal relations are applied:

$$
\left\{\begin{array}{l}
\int_{0}^{2 \pi} \sin [n \theta] \cos [m \theta] d \theta=0 \\
\int_{0}^{2 \pi} \cos [n \theta] \cos [m \theta] d \theta=\pi \delta_{m n} \\
\int_{0}^{2 \pi} \sin [n \theta] \sin [m \theta] d \theta=\pi \delta_{m n}
\end{array}\right.
$$

where $\delta_{m n}$ is the Kronecker delta symbol. 


\section{Case I: Annular plate with clamped boundary conditions}

We are now ready to derive the analytic solution of plate vibrations with clamped boundary condition for the continuous version of the MFS. The clamped boundary condition is defined by

$$
\left\{\begin{array}{l}
\mathbf{K}_{u}(u(\mathbf{x}))=0 \\
\mathbf{K}_{\theta}(u(\mathbf{x}))=0
\end{array}\right.
$$

Applying Eqs. (14a) \& (14b) on $\rho=\rho^{E} \& \rho=\rho^{I}$ and the fact that $A_{m}^{E}, B_{m}^{E}, C_{m}^{E}, D_{m}^{E}, A_{m}^{I}, B_{m}^{I}, C_{m}^{I}$, and $D_{m}^{I}$ are arbitrary constants, we obtain

$$
\begin{aligned}
& H_{m}^{(1)}\left(\lambda R^{E}\right) \times K_{m}\left(\lambda R^{E}\right) \times\left[J_{m}\left(\lambda R^{I}\right)-i \lambda J_{m}{ }^{\prime}\left(\lambda R^{I}\right)\right] \times\left[I_{m}\left(\lambda R^{I}\right)-i k I_{m}{ }^{\prime}\left(\lambda R^{I}\right)\right] \times \\
& \left|\begin{array}{llll}
\left.\mathbf{K}_{u}\left[J_{m}(\lambda \rho)\right]\right|_{\rho^{E}} & \left.\mathbf{K}_{u}\left[I_{m}(\lambda \rho)\right]\right|_{\rho^{E}} & \left.\mathbf{K}_{u}\left[H_{m}^{(1)}(\lambda \rho)\right]\right|_{\rho^{E}} & \left.\mathbf{K}_{u}\left[K_{m}(\lambda \rho)\right]\right|_{\rho^{E}} \\
\left.\mathbf{K}_{\theta}\left[J_{m}(\lambda \rho)\right]\right|_{\rho^{E}} & \left.\mathbf{K}_{\theta}\left[I_{m}(\lambda \rho)\right]\right|_{\rho^{E}} & \left.\mathbf{K}_{\theta}\left[H_{m}^{(1)}(\lambda \rho)\right]\right|_{\rho^{E}} & \left.\mathbf{K}_{\theta}\left[K_{m}(\lambda \rho)\right]\right|_{\rho^{E}} \\
\left.\mathbf{K}_{u}\left[J_{m}(\lambda \rho)\right]\right|_{\rho^{I}} & \left.\mathbf{K}_{u}\left[I_{m}\left(\lambda \rho^{I}\right)\right]\right|_{\rho^{I}} & \left.\mathbf{K}_{u}\left[H_{m}^{(1)}(\lambda \rho)\right]\right|_{\rho^{I}} & \left.\mathbf{K}_{u}\left[K_{m}(\lambda \rho)\right]\right|_{\rho^{I}} \\
\left.\mathbf{K}_{\theta}\left[J_{m}(\lambda \rho)\right]\right|_{\rho^{I}} & \left.\mathbf{K}_{\theta}\left[I_{m}\left(\lambda \rho^{I}\right)\right]\right|_{\rho^{I}} & \left.\mathbf{K}_{\theta}\left[H_{m}^{(1)}(\lambda \rho)\right]\right|_{\rho^{I}} & \left.\mathbf{K}_{\theta}\left[K_{m}(\lambda \rho)\right]\right|_{\rho^{I}}
\end{array}\right|=0
\end{aligned}
$$

where $\left.f(\rho, \theta)\right|_{\bar{\rho}}$ is defined to be $f(\bar{\rho}, \theta)$.

Since $H_{m}^{(1)}\left(\lambda R^{E}\right) \times K_{m}\left(\lambda R^{E}\right) \times\left[J_{m}\left(\lambda R^{I}\right)-i \lambda J_{m}^{\prime}\left(\lambda R^{I}\right)\right] \times\left[I_{m}\left(\lambda R^{I}\right)-i k I_{m}^{\prime}\left(\lambda R^{I}\right)\right] \neq 0$ for any real number $\lambda$, Eq. (18) can be further simplified to

$$
\left|\begin{array}{llll}
\left.\mathbf{K}_{u}\left[J_{m}(\lambda \rho)\right]\right|_{\rho^{E}} & \left.\mathbf{K}_{u}\left[I_{m}(\lambda \rho)\right]\right|_{\rho^{E}} & \left.\mathbf{K}_{u}\left[H_{m}^{(1)}(\lambda \rho)\right]\right|_{\rho^{E}} & \left.\mathbf{K}_{u}\left[K_{m}(\lambda \rho)\right]\right|_{\rho^{E}} \\
\left.\mathbf{K}_{\theta}\left[J_{m}(\lambda \rho)\right]\right|_{\rho^{E}} & \left.\mathbf{K}_{\theta}\left[I_{m}(\lambda \rho)\right]\right|_{\rho^{E}} & \left.\mathbf{K}_{\theta}\left[H_{m}^{(1)}(\lambda \rho)\right]\right|_{\rho^{E}} & \left.\mathbf{K}_{\theta}\left[K_{m}(\lambda \rho)\right]\right|_{\rho^{E}} \\
\left.\mathbf{K}_{u}\left[J_{m}(\lambda \rho)\right]\right|_{\rho^{I}} & \left.\mathbf{K}_{u}\left[I_{m}\left(\lambda \rho^{I}\right)\right]\right|_{\rho^{I}} & \left.\mathbf{K}_{u}\left[H_{m}^{(1)}(\lambda \rho)\right]\right|_{\rho^{I}} & \left.\mathbf{K}_{u}\left[K_{m}(\lambda \rho)\right]\right|_{\rho^{I}} \\
\left.\mathbf{K}_{\theta}\left[J_{m}(\lambda \rho)\right]\right|_{\rho^{I}} & \left.\mathbf{K}_{\theta}\left[I_{m}\left(\lambda \rho^{I}\right)\right]\right|_{\rho^{I}} & \left.\mathbf{K}_{\theta}\left[H_{m}^{(1)}(\lambda \rho)\right]\right|_{\rho^{I}} & \left.\mathbf{K}_{\theta}\left[K_{m}(\lambda \rho)\right]\right|_{\rho^{I}}
\end{array}\right|=0
$$

Equation (19) is just the eigenequation for annular plate with clamped boundary condition.

\section{Case II: Annular plate with simply supported boundary conditions}

The derivation of analytic solution for simply supported annular plate can be performed in a similar fashion. The simply supported boundary condition is defined by

$$
\left\{\begin{array}{l}
\mathbf{K}_{u}(u(\mathbf{x}))=0 \\
\mathbf{K}_{m}(u(\mathbf{x}))=0
\end{array}\right.
$$

Applying Eqs. (14a) \& (14c) and the fact that $A_{m}^{E}, B_{m}^{E}, C_{m}^{E}, D_{m}^{E}, A_{m}^{I}, B_{m}^{I}, C_{m}^{I}$, and $D_{m}^{I}$ are arbitrary, we obtain

$$
\begin{aligned}
& H_{m}^{(1)}\left(\lambda R^{E}\right) \times K_{m}\left(\lambda R^{E}\right) \times\left[J_{m}\left(\lambda R^{I}\right)-i \lambda J_{m}{ }^{\prime}\left(\lambda R^{I}\right)\right] \times\left[I_{m}\left(\lambda R^{I}\right)-i k I_{m}{ }^{\prime}\left(\lambda R^{I}\right)\right] \times \\
& \left|\begin{array}{llll}
\left.\mathbf{K}_{u}\left[J_{m}(\lambda \rho)\right]\right|_{\rho^{E}} & \left.\mathbf{K}_{u}\left[I_{m}(\lambda \rho)\right]\right|_{\rho^{E}} & \left.\mathbf{K}_{u}\left[H_{m}^{(1)}(\lambda \rho)\right]\right|_{\rho^{E}} & \left.\mathbf{K}_{u}\left[K_{m}(\lambda \rho)\right]\right|_{\rho^{E}} \\
\left.\mathbf{K}_{m}\left[J_{m}(\lambda \rho)\right]\right|_{\rho^{E}} & \left.\mathbf{K}_{m}\left[I_{m}(\lambda \rho)\right]\right|_{\rho^{E}} & \left.\mathbf{K}_{m}\left[H_{m}^{(1)}(\lambda \rho)\right]\right|_{\rho^{E}} & \left.\mathbf{K}_{m}\left[K_{m}(\lambda \rho)\right]\right|_{\rho^{E}} \\
\left.\mathbf{K}_{u}\left[J_{m}(\lambda \rho)\right]\right|_{\rho^{I}} & \left.\mathbf{K}_{u}\left[I_{m}\left(\lambda \rho^{I}\right)\right]\right|_{\rho^{I}} & \left.\mathbf{K}_{u}\left[H_{m}^{(1)}(\lambda \rho)\right]\right|_{\rho^{I}} & \left.\mathbf{K}_{u}\left[K_{m}(\lambda \rho)\right]\right|_{\rho^{I}} \\
\left.\mathbf{K}_{m}\left[J_{m}(\lambda \rho)\right]\right|_{\rho^{I}} & \left.\mathbf{K}_{m}\left[I_{m}\left(\lambda \rho^{I}\right)\right]\right|_{\rho^{I}} & \left.\mathbf{K}_{m}\left[H_{m}^{(1)}(\lambda \rho)\right]\right|_{\rho^{I}} & \left.\mathbf{K}_{m}\left[K_{m}(\lambda \rho)\right]\right|_{\rho^{I}}
\end{array}\right|=0
\end{aligned}
$$

which can be further simplified to

$$
\left|\begin{array}{llll}
\left.\mathbf{K}_{u}\left[J_{m}(\lambda \rho)\right]\right|_{\rho^{E}} & \left.\mathbf{K}_{u}\left[I_{m}(\lambda \rho)\right]\right|_{\rho^{E}} & \left.\mathbf{K}_{u}\left[H_{m}^{(1)}(\lambda \rho)\right]\right|_{\rho^{E}} & \left.\mathbf{K}_{u}\left[K_{m}(\lambda \rho)\right]\right|_{\rho^{E}} \\
\left.\mathbf{K}_{m}\left[J_{m}(\lambda \rho)\right]\right|_{\rho^{E}} & \left.\mathbf{K}_{m}\left[I_{m}(\lambda \rho)\right]\right|_{\rho^{E}} & \left.\mathbf{K}_{m}\left[H_{m}^{(1)}(\lambda \rho)\right]\right|_{\rho^{E}} & \left.\mathbf{K}_{m}\left[K_{m}(\lambda \rho)\right]\right|_{\rho^{E}} \\
\left.\mathbf{K}_{u}\left[J_{m}(\lambda \rho)\right]\right|_{\rho^{I}} & \left.\mathbf{K}_{u}\left[I_{m}\left(\lambda \rho^{I}\right)\right]\right|_{\rho^{I}} & \left.\mathbf{K}_{u}\left[H_{m}^{(1)}(\lambda \rho)\right]\right|_{\rho^{I}} & \left.\mathbf{K}_{u}\left[K_{m}(\lambda \rho)\right]\right|_{\rho^{I}} \\
\left.\mathbf{K}_{m}\left[J_{m}(\lambda \rho)\right]\right|_{\rho^{I}} & \left.\mathbf{K}_{m}\left[I_{m}\left(\lambda \rho^{I}\right)\right]\right|_{\rho^{I}} & \left.\mathbf{K}_{m}\left[H_{m}^{(1)}(\lambda \rho)\right]\right|_{\rho^{I}} & \left.\mathbf{K}_{m}\left[K_{m}(\lambda \rho)\right]\right|_{\rho^{I}}
\end{array}\right|=0
$$

Equation (22) is the eigenequation for simply supported annular plate. 


\section{Case III: Annular plate with free boundary conditions}

The above procedure for the derivation of analytic solution can also be extended to free boundary condition, which is defined by

$$
\left\{\begin{array}{l}
\mathbf{K}_{m}(u(\mathbf{x}))=0 \\
\mathbf{K}_{v}(u(\mathbf{x}))=0
\end{array}\right.
$$

Applying Eqs. (14c) and (14d), we can obtain the final eigenequation by a similar procedure:

$$
\left|\begin{array}{llll}
\left.\mathbf{K}_{m}\left[J_{m}(\lambda \rho)\right]\right|_{\rho^{E}} & \left.\mathbf{K}_{m}\left[I_{m}(\lambda \rho)\right]\right|_{\rho^{E}} & \left.\mathbf{K}_{m}\left[H_{m}^{(1)}(\lambda \rho)\right]\right|_{\rho^{E}} & \left.\mathbf{K}_{m}\left[K_{m}(\lambda \rho)\right]\right|_{\rho^{E}} \\
\left.\mathbf{K}_{v}\left[J_{m}(\lambda \rho)\right]\right|_{\rho^{E}} & \left.\mathbf{K}_{v}\left[I_{m}(\lambda \rho)\right]\right|_{\rho^{E}} & \left.\mathbf{K}_{v}\left[H_{m}^{(1)}(\lambda \rho)\right]\right|_{\rho^{E}} & \left.\mathbf{K}_{v}\left[K_{m}(\lambda \rho)\right]\right|_{\rho^{E}} \\
\left.\mathbf{K}_{m}\left[J_{m}(\lambda \rho)\right]\right|_{\rho^{I}} & \left.\mathbf{K}_{m}\left[I_{m}\left(\lambda \rho^{I}\right)\right]\right|_{\rho^{I}} & \left.\mathbf{K}_{m}\left[H_{m}^{(1)}(\lambda \rho)\right]\right|_{\rho^{I}} & \left.\mathbf{K}_{m}\left[K_{m}(\lambda \rho)\right]\right|_{\rho^{I}} \\
\left.\mathbf{K}_{v}\left[J_{m}(\lambda \rho)\right]\right|_{\rho^{I}} & \left.\mathbf{K}_{v}\left[I_{m}\left(\lambda \rho^{I}\right)\right]\right|_{\rho^{I}} & \left.\mathbf{K}_{v}\left[H_{m}^{(1)}(\lambda \rho)\right]\right|_{\rho^{I}} & \left.\mathbf{K}_{v}\left[K_{m}(\lambda \rho)\right]\right|_{\rho^{I}}
\end{array}\right|=0
$$

Equation (24) is the eigenequation for free circular plate.

It is noted that the resulting eigenequations of Eqs. (19), (22), and (24), derived from the continuous version of MFS, for the three different boundary conditions of the annular plate are just the exact solutions of the problem. The first few eigenvalues for the three cases $\left(\rho^{E}=1.0, \rho^{I}=0.5\right.$, and $\left.v=0.3\right)$ are shown in Table $1 \sim$ Table 3 and will be used to validate the numerical results obtained in the next section.

Table 1 The eigenvalues $(\lambda)$ for the clamped annular plate $\left(\rho^{E}=1.0, \rho^{I}=0.5\right.$, and $\left.\nu=0.3\right)$

\begin{tabular}{|c|c|c|c|c|c|c|c|}
\hline \multirow{2}{*}{$m$} & \multicolumn{9}{|c|}{ eigenvalues for $n$ of } \\
\cline { 2 - 9 } & 0 & 1 & 2 & 3 & 4 & 5 & 6 \\
\hline 1 & 9.45 & 9.50 & 9.66 & 9.95 & 10.37 & 10.94 & 11.64 \\
\hline 2 & 15.70 & 15.74 & 15.87 & 16.10 & 16.42 & 16.83 & 17.33 \\
\hline
\end{tabular}

where $m$ refers to the number of nodal circles and $\mathrm{n}$ is the number of nodal diameters.

Table 2 The eigenvalues $(\lambda)$ for the simply supported annular plate $\left(\rho^{E}=1.0, \rho^{I}=0.5\right.$, and $\left.v=0.3\right)$

\begin{tabular}{|c|c|c|c|c|c|c|c|}
\hline \multirow{2}{*}{$m$} & \multicolumn{7}{|c|}{ eigenvalues for $n$ of } \\
\cline { 2 - 9 } & 0 & 1 & 2 & 3 & 4 & 5 & 6 \\
\hline 1 & 6.33 & 6.47 & 6.86 & 7.48 & 8.27 & 9.18 & 10.17 \\
\hline 2 & 12.60 & 12.67 & 12.90 & 13.27 & 13.77 & 14.39 & 15.12 \\
\hline 3 & 18.87 & 18.92 & 19.08 & 19.33 & 19.69 & & \\
\hline
\end{tabular}

where $m$ refers to the number of nodal circles and $\mathrm{n}$ is the number of nodal diameters.

Table 3 The eigenvalues $(\lambda)$ for the free annular plate $\left(\rho^{E}=1.0, \rho^{I}=0.5\right.$, and $\left.\nu=0.3\right)$

\begin{tabular}{|c|c|c|c|c|c|r|r|}
\hline \multirow{2}{*}{$m$} & \multicolumn{9}{|c|}{ eigenvalues for $n$ of } \\
\cline { 2 - 9 } & 0 & 1 & 2 & 3 & 4 & 5 & 6 \\
\hline 1 & & & 2.07 & 3.38 & 4.59 & 5.74 & 6.86 \\
\hline 2 & 3.05 & 4.15 & 5.58 & 6.89 & 8.17 & 9.45 & 10.75 \\
\hline 3 & 9.61 & 9.81 & 10.37 & 11.16 & 12.10 & 13.12 & 14.21 \\
\hline 4 & 15.79 & 15.89 & 16.19 & 16.66 & 17.27 & 18.00 & 18.81 \\
\hline
\end{tabular}

where $m$ refers to the number of nodal circles and $\mathrm{n}$ is the number of nodal diameters.

\section{NUMERICAL RESULTS}

In this section, three numerical experiments are carried out for the discrete version of the MFS and results are discussed and compared with the analytical solutions obtained in the last section. Since all these three cases have exact solutions, we can validate our numerical valuations by the discrete version of the MFS. Numerical eigenvalues of these three problems are insensitive with respect to numbers of nodes and locations of source points. 


\section{Case I: Annular plate with clamped boundary conditions}

An annular plate with exterior and interior radii, $\rho^{E}=$ $1.0 \& \rho^{I}=0.5$, and $v=0.3$ subjected to clamped boundary condition is considered. The schematic of boundary field and source points are described in Fig. 2. On the other hand, Fig. 3 shows the results of the direct determinant search method for different numbers of nodes and locations of sources. In Fig. 3 , the value $\lambda$, in which there is a cusp, is an eigenvalue since the corresponding determinant value is suddenly minified [13]. The numerical eigenvalues are in good agreement (up to 0.01 ) with the exact solutions as shown in Table 1. Also, the results demonstrate that the MFS is insensitive with respect to the locations of source points. It is noticed that there is no spurious eigenvalues as we have studied analytically in the last section.

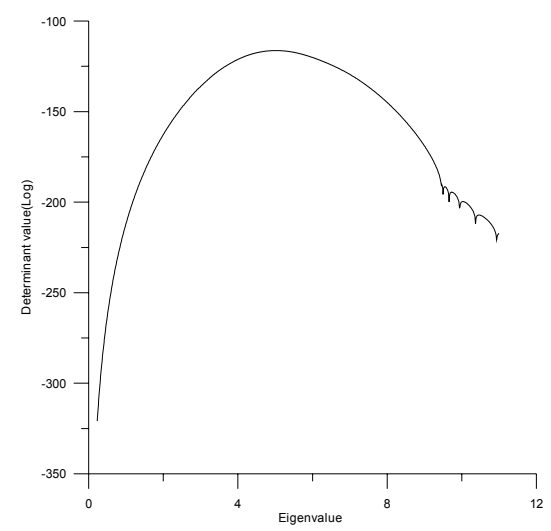

(a) full range of eigenvalue

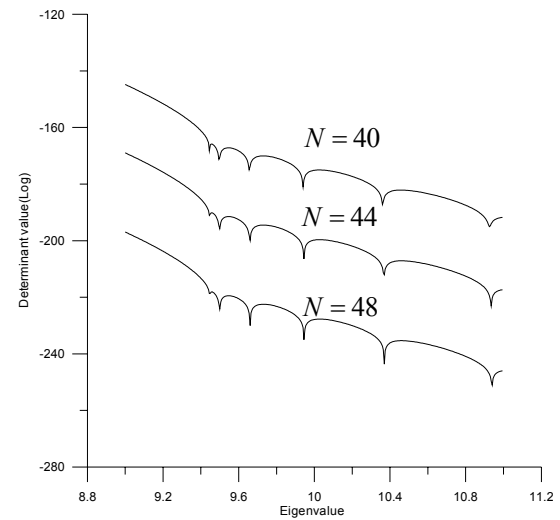

(b) different numbers of nodes $\left(R^{E}=2.4, R^{I}=0.3\right)$

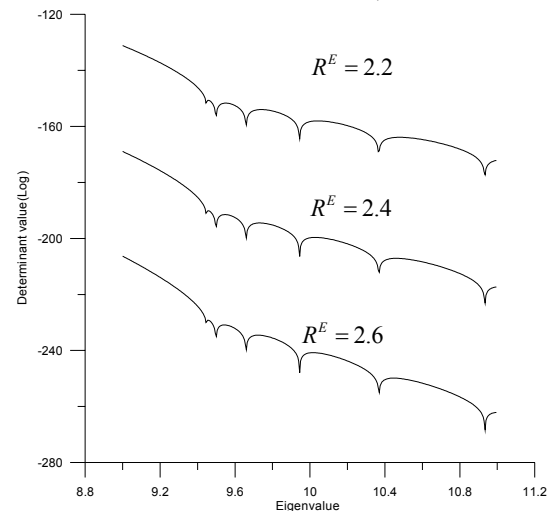

(c) different $R^{E}\left(\mathrm{~N}=44, R^{I}=0.3\right)$

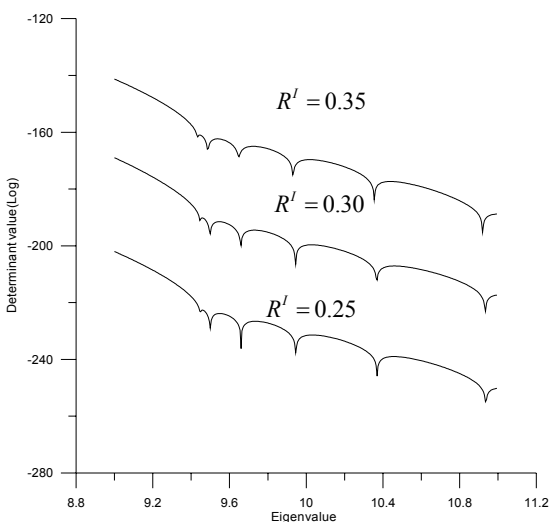

(d) different $R^{I}\left(\mathrm{~N}=44, R^{E}=2.4\right)$

Fig. 3 Results of the direct determinant search method for clamped annular plate

\section{Case II: Annular plate with simply supported boundary conditions}

We next consider the case of simply supported boundary condition, for $\rho^{E}=1.0, \rho^{I}=0.5$, and $\nu=0.3$. Figure 4 depicts the results of the direct determinant search method for different number of nodes and locations of sources. The numerical eigenvalues are also accurate up to 0.01 with the exact solutions shown in Table 2 and no spurious eigenvalues are observed. In these figures, it is convinced that the MFS can provide accurate solutions with few nodes $(\mathrm{N}=40,44,48)$ and be insensitive with respect to the locations of source points.

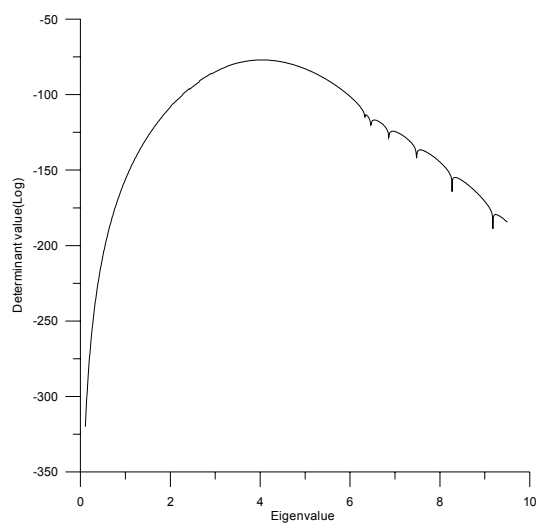

(a) full range of eigenvalue

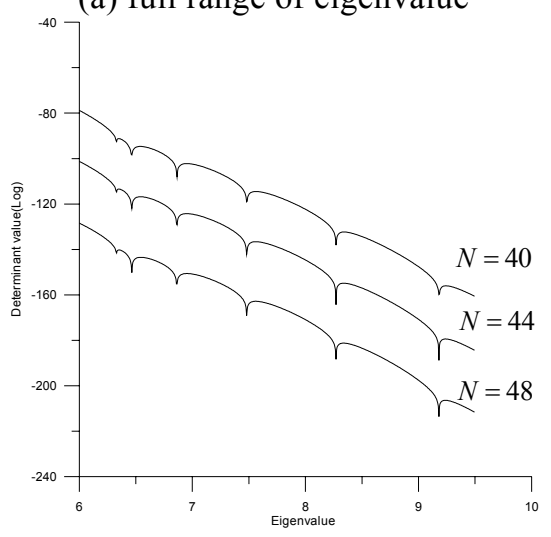

(b) different numbers of nodes $\left(R^{E}=3.0, R^{I}=0.3\right)$ 


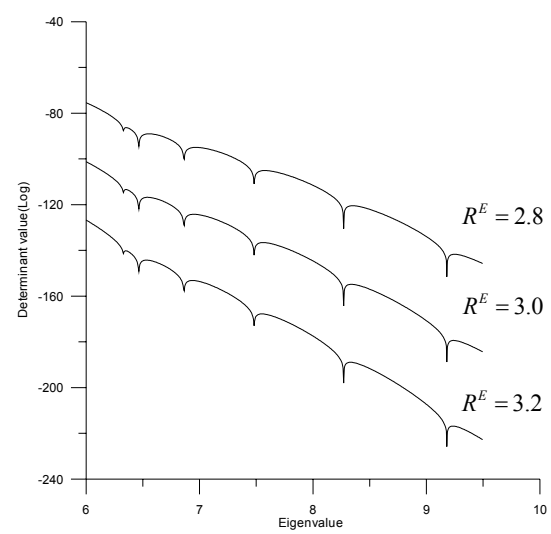

(c) different $R^{E}\left(\mathrm{~N}=44, R^{I}=0.3\right)$

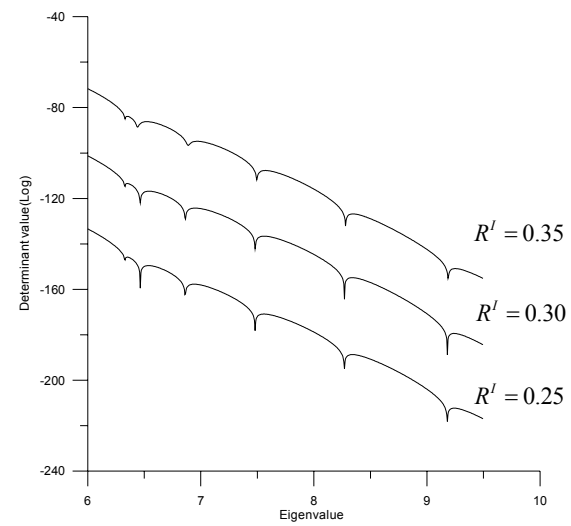

(d) different $R^{I}\left(\mathrm{~N}=44, R^{E}=3.0\right)$

Fig. 4 Results of the direct determinant search method for simply supported annular plate

\section{Case III: Annular plate with free boundary conditions}

The application of MFS scheme for the annular plate with free boundary condition is straightforward. Here we also choose $\rho^{E}=1.0, \rho^{I}=0.5$, and $v=0.3$. In Fig. 5 , we show the results of the direct determinant search method for different number of nodes and locations of sources. The numerical eigenvalues are agree (up to 0.01 ) with the exact solutions as shown in Table 3 . In Fig. 5, no spurious eigenvalues is observed and the results reveal that the MFS performs well for different locations of sources.

From the above numerical results, it is found that more nodes are required in order to capture all true eigenvalues for the case of free boundary condition than the simply supported and clamped boundary conditions (free $>$ simply supported $>$ clamped). Similarly, it is desirable to have farther source point locations for the free boundary condition than the other two boundary conditions (free $>$ simply supported $>$ clamped). This is due to the reason that for the eigenvalue problem higher accuracy is required for plate vibrations with free boundary condition than simply supported and clamped boundary conditions according to the physics of free vibration of plates.

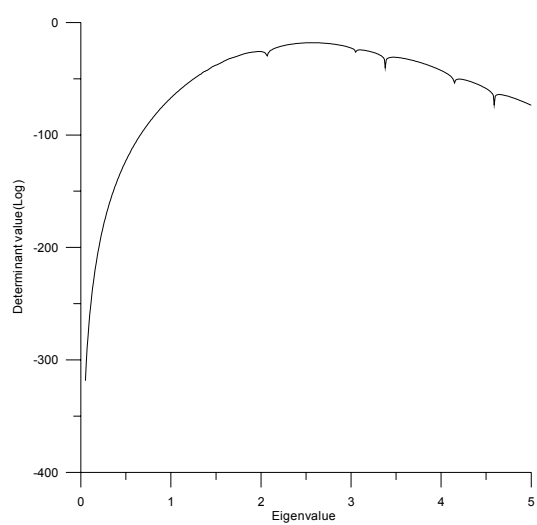

(a) full range of eigenvalue

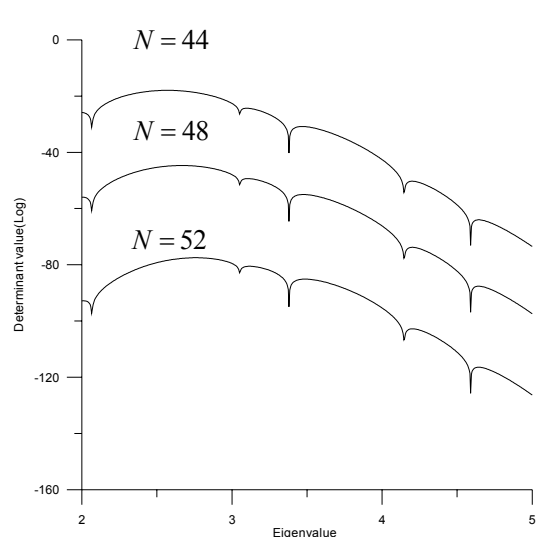

(b) different numbers of nodes $\left(R^{E}=5.0, R^{I}=0.29\right)$

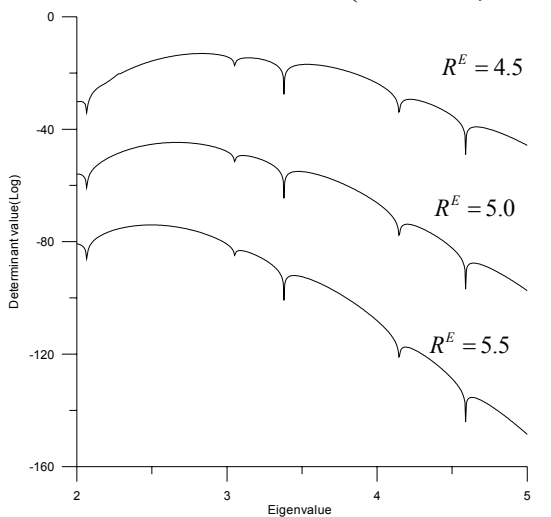

(c) different $R^{E}\left(\mathrm{~N}=48, R^{I}=0.29\right)$

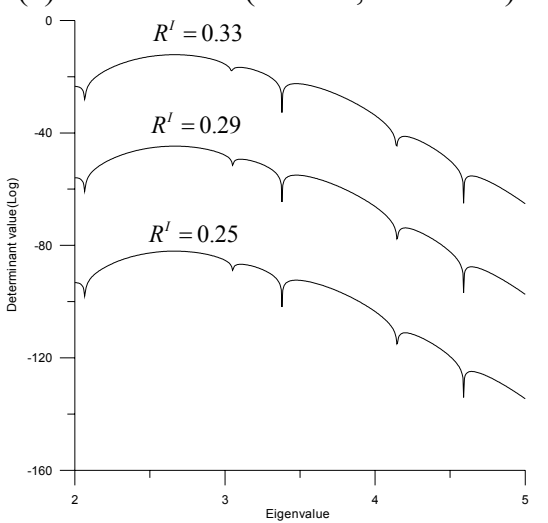

(d) different $R^{I}\left(\mathrm{~N}=48, R^{E}=5.0\right)$

Fig. 5 Results of the direct determinant search method for free annular plate 


\section{CONCLUSIONS}

An analytical study is carried out to demonstrate that if the complex valued MFS combined with the mix potential method is applied to solve plate vibrations in multiply connected domains, then there is no spurious eigenvalue. Numerical experiments are also consistent with the above statement. The direct determinant search method is utilized to obtain the eigenvalues, with the results showing good agreement with the exact solutions for very few nodes. This implies that the MFS is a very efficient numerical method. Moreover, numerical experiments also indicate that the MFS is insensitive to the locations of source points. According to our numerical studies, it is also found that more and farther source points are necessary for the free boundary condition than the simply supported and clamped boundary conditions. Therefore, it is convinced that the MFS is a meshless numerical method free from meshes, singularities, numerical integrations, and treatment of spurious eigenvalues. Thus, through this work we have demonstrated the simplicity and efficiency of the MFS to solve plate vibrations in multiply connected domains.

\section{ACKNOWLEDGEMENTS}

The support under Grant NSC 94-2211-E-464-003 by the National Science Council of Taiwan is gratefully acknowledged.

\section{REFERENCES}

1. Kupradze, V. D. and Aleksidze, M. A., "The Method of Functional Equations for the Approximate Solution of Certain Boundary Value Problem," USSR Computational Mathematics and Mathematical Physics, 4, pp. 82-126 (1964).

2. Fairweather, G. and Karageorghis, A., "The Method of Fundamental Solutions for Elliptic Boundary Value Problems," Advances in Computational Mathematics, 9, pp. 69-95 (1998).

3. Golberg, M. A. and Chen, C. S., "The Method of FundaMental Solutions for Potential, Helmholtz and Diffusion Problems," Boundary Integral Methods: Numerical and Mathematical Aspects, Golberg, M. A., Ed., pp. 103-176 (1998).

4. Fairweather, G., Karageorghis, A. and Martin, P. A., "The Method of Fundamental Solutions for Scattering and Radiation Problems," Engineering Analysis with Boundary Elements, 27, pp. 759-769 (2003).

5. Cho, H. A., Golberg, M. A., Muleshkov, A. S. and Li, X., "Trefftz Methods for Time Dependent Partial Differential Equations," CMC: Computers, Materials, and Continua, 1, pp. 1-37 (2004).

6. Young, D. L., Tsai, C. C. and Fan, C. M., "Direct Approach to Solve Nonhomogeneous Diffusion Problems Using Fundamental Solutions and Dual Reciprocity
Methods," Journal of the Chinese Institute of Engineers, 27, pp. 597-609 (2004).

7. Young, D. L., Tsai, C. C., Murugesan, K., Fan, C. M. and Chen, C. W., "Time-Dependent Fundamental Solutions for Homogeneous Diffusion Problems," Engineering Analysis with Boundary Elements, 28, pp. 1463-1473 (2004).

8. Hu, S. P., Fan, C. M., Chen, C. W. and Young, D. L., "Method of Fundamental Solutions for Stokes' First and Second Problems," Journal of Mechanics, 21, pp. 25-32 (2005).

9. Tsai, C. C., Young, D. L., Chen, C. W. and Fan, C. M., "The Method of Fundamental Solutions for Eigenproblems in Domains with and Without Interior Holes," Proceedings of the Royal Society of London, Series A, 462, pp. 1443-1466 (2006).

10. Leissa, A. W., Laura, P. A. A. and Gutierrez, R. H., "Transverse Vibrations of Circular Plates Having NonUniform Edge Constraints," Journal of Acoustical Society of American, 66, pp. 180-184 (1979).

11. Kitahara, M., Boundary Integral Equation Methods in Eigenvalue Problems of Elastodynamics and Thin Plates, Elsevier, Amsterdam (1985).

12. Hutchinson, J. R., "Vibration of Plates," Boundary Elements X, Brebbia, C. A., Ed., Springer, Berlin (1988).

13. Chen, J. T., Lin, S. Y., Chen, K. H. and Chen, I. L., "Mathematical Analysis and Numerical Study of True and Spurious Eigenequations for Free Vibration of Plates Using Real-part BEM," Computational Mechanics, 34, pp. 165-180 (2004).

14. Kondapalli, P. S. and Shippy, D. J., "Analysis of Acoustic Scattering in Fluids and Solids by the Method of Fundamental Solutions," Journal of Acoustical Society of American, 91, pp. 1844-1854 (1992).

15. Kang, S. W. and Lee, J. M., "Application of Free Vibration Analysis of Membranes Using the Non-Dimensional Dynamic Influence Function," Journal of Sound and Vibration, 234, pp. 455-470 (2000).

16. Chen, J. T., Kou, S. R., Chen, K. H. and Cheng, Y. C., "Comments on 'Vibration Analysis of Arbitrary Shaped Membranes Using the Non-Dimensional Dynamic Influence Function'," Journal of Sound and Vibration, 235, pp. 156-171 (2000).

17. Chen, J. T., Chang, M. H., Chen, K. H. and Lin, S. R., "The Boundary Collocation Method with Meshless Concept for Acoustic Eigenanalysis of Two-Dimensional Cavities Using Radial Basis Function," Journal of Sound and Vibration, 257, pp. 667-711 (2002).

18. Chen, J. T., Chang, M. H., Chen, K. H. and Chen, I. L., "Boundary Collocation Method for Acoustic Eigenanalysis of Three-Dimensional Cavities Using Radial Basis Function," Computational Mechanics, 29, pp. 392-408 (2002).

19. Kang, S. W. and Lee, J. M., "Free Vibration Analysis of Arbitrary Shaped Plates with Clamped Edges Using Wave-Type Functions," Journal of Sound and Vibration, 242, pp. 9-26 (2001).

20. Karageorghis, A., "The Method of Fundamental Solutions for the Calculation of the Eigenvalues of the Helmholtz Equation," Applied Mathematics Letters, 14, pp. 837-842 (2001). 
21. Young, D. L., Hu, S. P., Chen, C. W., Fan, C. M. and Murugesan, K., "Analysis of Elliptical Waveguides by Method of Fundamental Solutions," Microwave and Optical Technology Letters, 44, pp. 552-558 (2005).

22. Chen, C. W., Fan, C. M., Young, D. L., Murugesan, K. and Tsai, C. C., "Eigenanalysis for Membranes with Stringers Using the Methods of Fundamental Solutions and Domain Decomposition," CMES: Computer Modeling in Engineering and Sciences, 8, pp. 29-44 (2005).

23. Burton, A. J. and Miller, G. F., "The Application of Integral Equation Methods to the Numerical Solution of Some Exterior Boundary-Value Problems," Proceeding of the Royal Society A, 323, pp. 201-210 (1971).

24. Panich, O. I., "On the Question of the Solvability of the Exterior Boundary Problem for the Wave Equation and Maxwell's Equation," Uspekhi Matematicheskikh Nauk, 20, pp. 221-226 (1965).
25. Brakhage, H. and Werner, P., "Über das Dirichletsche Aussenraumproblem Für Die Helmholtzsche Schwingungsgleichung," Archiv Der Mathematik, 16, pp. 325-329 (1965).

26. Tai, G. R. G. and Shaw, R. P., "Helmholtz Equation Eigenvalues and Eigenmodes for Arbitrary Domains," Journal of Acoustical Society of American, 56, pp. 796-804 (1974).

27. Chen, J. T., Liu, L. W. and Hong, H. K., "Spurious and True Eigensolutions of Helmholtz BIEs and BEMs for a Multiply Connected Problem," Proceeding of the Royal Society Series A, 459, pp. 1897-1924 (2003).

(Manuscript received December 16, 2005, accepted for publication June 26, 2006.) 
